\title{
ON SENSITIVITY TO GLARE AND ITS RELATIONSHIP WITH MACULAR PIGMENT
}

\author{
Sneha Jain ${ }^{1}$, Jan Wienold ${ }^{1}$, Marilyne Andersen ${ }^{1}$ \\ ${ }^{1}$ École polytechnique fédérale de Lausanne, Lausanne, SWITZERLAND \\ sneha.jain@epfl.ch
}

\begin{abstract}
Current trends in discomfort glare research have suggested the influence of physiological parameters on individual glare perception. To this end, we hypothesize that a specific ocular physiology characteristic, namely the macular pigment (MP) in the retina, could have an influence on glare sensitivity, encouraged by recent findings from the literature that have shown that high MP levels were indicative of better visual performance. This study investigates whether a person's sensitivity to glare could be somehow correlated to their macular pigment optical density (MPOD). We measured MPOD in 56 participants and compared it with their discomfort glare thresholds, which were determined psychophysically by exposing the participants to a series of lighting conditions varying in intensity. We found that the influence of MPOD on glare sensitivity is borderline significant with small effect size but does not follow intuition. Additional data will be required to validate and refine these initial findings.
\end{abstract}

\section{Keywords: Glare, Macular pigment, User assessment, Sensitivity}

\section{Introduction and state of the art}

Properly addressing glare risks in buildings is crucial towards achieving comfortable visual environments. It is, therefore, necessary to understand what causes or influences the perception of discomfort glare. Although several glare prediction models based on physical quantities have been developed in the last two decades, current models are unable to capture the large inter-individual variability that is observed in the perception of discomfort glare (EbleHankins and Waters, 2005). If we assume that the tolerance towards discomfort glare does indeed vary among individuals, it seems plausible that certain eye morphology parameters could at least in part explain this variability.

Previous studies have shown the influence of macular pigment on the visual performance in the presence of glare (Lien and Hammond, 2011; Stringham et al., 2011, 2010; Wenzel et al., 2006). The yellow macular pigments, mainly comprised of carotenoids (lutein, zeaxanthin, and mesozeaxanthin), are deposited in the fovea in the Henle fibre layer, in the parafovea and in the inner plexiform layers of the retina. Macular pigment covers about $6.7^{\circ}$ in the horizontal and $3.0^{\circ}$ in the vertical directions of the central retina (Bernstein et al., 2010). Several techniques are available to measure macular pigment that mainly include either physical methods (such as Fundus autofluorescence imaging (Delori et al., 2001)) or psychophysical methods (such as heterochromatic flicker photometry (HFP) (van der Veen et al., 2009)). In visual comfort research, psychophysical methods, mainly HFP, is used more often to measure macular pigment optical density (MPOD). MPOD is a measurement of the attenuation of blue light by macular pigment and is linearly related to the amount of lutein and zeaxanthin in the macula (Bernstein et al., 2010).

A study by Stringham et.al (Stringham et al., 2004) indicates that a high MPOD level protects the retina as the macular pigments absorb high-energy short-wavelength light. Another study from the same author showed that subjects with broader MPOD spatial distribution would perceive less discomfort glare (Stringham et al., 2011). It was hypothesized from this study that the subjects with relatively high macular pigment density tolerate more intense light. A study by Lien et al. (Lien and Hammond, 2011) also indicated that the absorption of short wavelength light by the macular pigment reduces the discomfort glare. However, another study from Loughman et al. (Loughman et al., 2010) concluded that visual performance under glare conditions were unrelated to macular pigment. The lack of consistency between these findings suggests a need for more research in this area. 
To address this need, the present study discusses preliminary results from a test room experiment conducted with 56 human participants to evaluate the glare sensitivity among the participants and its relationship with macular pigment density measured using psychophysical methods.

\section{Method}

A total of 56 young healthy individuals between 18 and 35 years of age participated in the study. The requirements for selection were to be in healthy conditions, have a normal colour vision, no other visual impairment, have a BMI between normal ranges, must have English proficiency level $\mathrm{C} 1$ or higher, must not use drugs and must not abuse of alcohol. Experiments were conducted at EPFL campus in Lausanne, Switzerland in a controlled test room environment with no daylight access in which the thermal and visual parameters of the room were monitored. The experiments were approved by the EPFL Human Research Ethics Committee (No. 0652019).

\subsection{Experimental design and setup}

\subsubsection{Measurement of visual characteristics}

We measured the best corrected visual acuity and contrast sensitivity of each participant monocularly to collect baseline ocular data and ensure normal vision. These tests were performed on a validated computer software FrACT 3.10 .5 (Bach, 1996) at a distance of $170 \mathrm{cms}$ from the computer screen. Calibration settings were adapted as per user's computer screen resolution and the distance between user's eyes and the screen were set to allow the measure of the maximal acuity.

Macular pigment optical density (MPOD) was measured using a macular pigment screener device, QuantifEye MPS II, that uses the heterochromatic flicker photometry method to provide an estimate of the blue light absorption of MP. MPOD values are measured on a scale of 0 to 1 , where a lower value indicates a higher level of blue light hitting the macula. In this test, participants viewed a centrally fixated target and made flicker matches at two light wavelengths of $465 \mathrm{~nm}$ (blue light) which is absorbed by the MP and another of $530 \mathrm{~nm}$ (green light), not absorbed by MP. Flicker matches were made in the foveal region of the retina. These measurements were done under electric lighting.

\subsubsection{Glare sensitivity test setup}

Participants' sensitivity to glare was evaluated experimentally in that they were asked to rate glare from a dimmable electric light with predefined levels of luminance that the participants were exposed to in their near foveal field of view. The LED glare source was of diameter $10 \mathrm{~cm}$, CCT of $4800 \mathrm{~K}$ with a diffuser sheet, and the angle between the glare source and the participant's line of sight was $20^{\circ}$. Note that the glare source was the only light source in the test room during the glare assessment session. Participants were exposed to 5 light levels that differed in glare stimuli luminance ranging from $265363 \mathrm{~cd} / \mathrm{m} 2(U G R=36)$ to $2000 \mathrm{~cd} / \mathrm{m} 2$ (UGR $=8$ ) designed to cover the glare sensation spectrum from imperceptible (UGR < 10) to intolerable glare (UGR > 34) as shown in Table 1. The naming convention followed for the scenes shown to the participants is based on the percentage of lighting intensity of the LED. These five light levels are illustrated in Figure 1 as false colour images and the glare scores and luminance values associated with them are provided in Table 1.

As illustrated in Figure 2, each participant was exposed to a sequence of nine visual scenes in total, reordering and sometimes repeating the five light scenarios described above as follows: the first three scenes, which varied from lowest $(1 \%)$, middle $(10 \%)$ and highest $(100 \%)$ light intensity, were the training scenes and were thus not randomized between the participant to avoid anchor bias (Fotios, 2009); the remaining six visual scenes consisted of the five testing scenes considered in the analysis and were thus randomized to avoid order bias and included one repeated condition to check the consistency of the evaluation done by the participant. One sequence is shown as an example in Figure 2. To further check the reliability of the test and consistency of the participants, a post-trial session was then conducted where participants were exposed to continuously increasing intensity of the LED until they reported visual discomfort and the intensity value was recorded. 


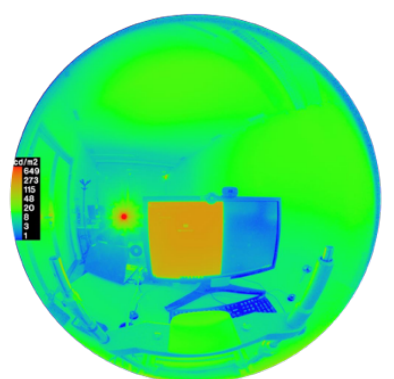

Light level $100 \%$

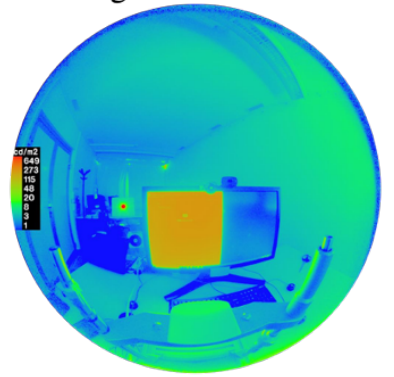

Light level 5\%

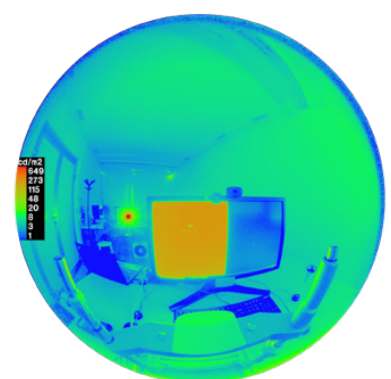

Light level 25\%

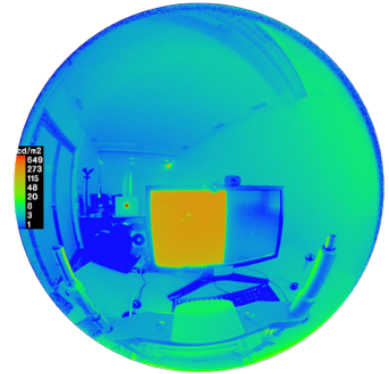

Light level 1\%

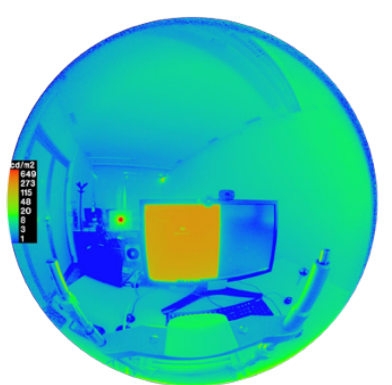

Light level 10\%

Figure $1 \mathrm{HDR}$ false colour images of the experimental scenarios shown to participants

Training scenes

Order not randomized

\begin{tabular}{|l|l|l|}
\hline Scene 1 & Scene 2 & Scene 3 \\
\hline $1 \%$ & $10 \%$ & $100 \%$ \\
\hline
\end{tabular}

Test scenes

Order randomized

\begin{tabular}{l:l:l:l:l:l:}
\hline Scene 1 & Scene 2 & Scene 3 & Scene 4 & Scene 5 & Scene 6 \\
\hdashline $5 \%$ & $10 \%$ & $5 \%$ & $25 \%$ & $100 \%$ & $1 \%$ \\
\hline
\end{tabular}

Figure 2 Experimental scenes shown to the participants

Table 1 Description of the visual properties of the five experimental conditions

\begin{tabular}{|l|l|l|l|l|}
\hline Scene & UGR & $\begin{array}{l}\text { Source } \\
\text { Luminance } \\
\left(\mathbf{c d} / \mathbf{m}^{2}\right)\end{array}$ & CGI & $\begin{array}{l}\text { Subjective } \\
\text { Categorization (as per } \\
\text { (Carlucci et al., 2015) }\end{array}$ \\
\hline $1 \%$ & 9 & 2019 & 5 & Imperceptible \\
\hline $5 \%$ & 15 & 12037 & 18 & Perceptible \\
\hline $10 \%$ & 20 & 25686 & 22 & Unacceptable \\
\hline $25 \%$ & 26 & 66051 & 29 & Uncomfortable \\
\hline $100 \%$ & 35 & 265636 & 37 & Intolerable \\
\hline
\end{tabular}

\subsection{Experimental procedure and questionnaires}

The experimental procedure lasted for 30 minutes with one participant at a time and was sequenced according to the timeline shown in Figure 3. For each session, participants were first introduced to the experiment and invited to sign the consent form. They then completed a 
background questionnaire which allowed to collect their baseline data: questions were about their age, gender, eye colour, their current mood, feelings and physical state, and their sensitivity and preferences towards certain indoor environmental parameters such as heat, cold, bright light, view to the outdoors. These questions were included to evaluate potential confounding factors, if any.

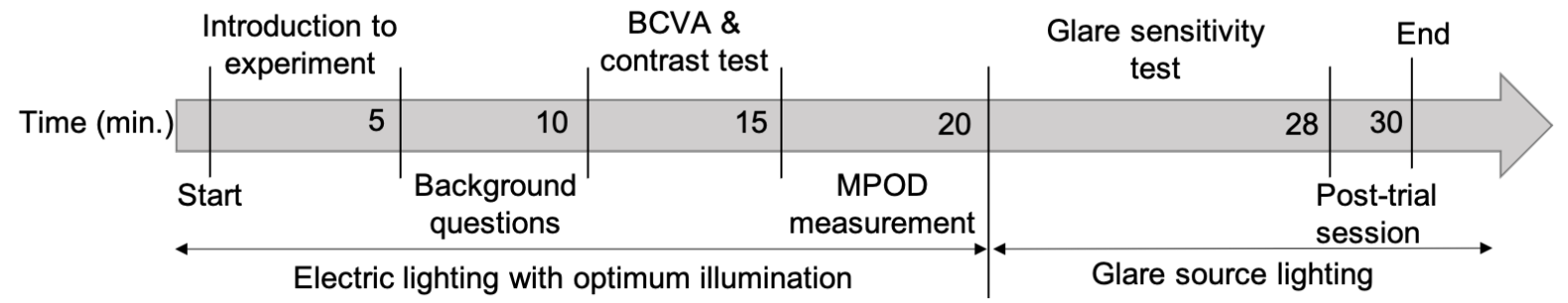

Figure 3 Experiment timeline

After completing the background survey, participants performed a visual acuity and contrast test on computer screen. Then, the MPOD for participant's left and right eye were measured using the MPSII screener device. Afterwards, the glare sensitivity test was conducted in the setup described in section 2.1.2. Participants performed the test with their chin fixed on a chin rest to keep their head in a constant position with respect to the computer screen and the glare source. During this test, the participants kept their focus on the screen, while the glare source was at $20^{\circ}$ horizontal and $0^{\circ}$ vertical from their central line of the sight and visible in their parafoveal field of view. Participants answered question 1 and 2 shown in Table 2 at the start of the test. Then, they were shown 9 scenes of varying glare source intensities as described in section 2.1.2. During the exposure to each scene for 20 seconds, participants read the text shown on the screen. After 20 seconds, they were asked to rate the discomfort from glare on a binary Yes/No scale and on a 6-point Likert scale listed in Table 2 as questions 3 and 4 respectively. We chose two glare scales to compare the responses and ensure consistency in within-subject responses. We selected a 6-pt scale to represent all the glare levels and avoid ambiguity created by a neutral middle point in an odd number scale. At the end of the procedure, the post-trial session was conducted where participants reported the threshold intensity causing discomfort from the glare.

Table 2 Questionnaires asked during glare sensitivity test

\begin{tabular}{|c|c|}
\hline Survey questions & Response scale \\
\hline $\begin{array}{l}\text { 1. Are you sensitive to bright light in } \\
\text { general? }\end{array}$ & Yes - No - I don't know \\
\hline $\begin{array}{l}\text { 2. Please rate your sensitivity to bright } \\
\text { light: }\end{array}$ & $\begin{array}{l}\text { Not sensitive } 0-1-2-3-4-5-6-7-8- \\
9-10 \text { Very sensitive }\end{array}$ \\
\hline $\begin{array}{l}\text { 3. Do you feel any discomfort due to } \\
\text { glare at the moment? }\end{array}$ & Yes - No \\
\hline $\begin{array}{l}\text { 4. How much discomfort are you } \\
\text { experiencing from the glare at the } \\
\text { moment? }\end{array}$ & $\begin{array}{l}\text { Not at all - Slight - Moderate - } \\
\text { Strong - Very Strong - Extreme }\end{array}$ \\
\hline
\end{tabular}

\subsection{Data cleansing and statistical methods}

Data collected was checked thoroughly to ensure consistency in within-subject responses. For any participant, if the difference in glare rating on the 6-point scale was more than 1-point for the repeated light intensity, then the session was removed from the final analysis (which happened for 3 participants). 
Descriptive analysis was performed to compare the subjective responses to the varying luminance of the glare source reported on the binary and the 6-pt scale. We used Spearman's rank correlation to compare the glare source luminance with the subjective responses reported on the 6-pt scale.

We derived a glare threshold for each participant from their subjective responses reported during the glare sensitivity test, to categorize the participants as less or more sensitive to glare. The light intensity at which a participant's discomfort glare rating changes from 'No' discomfort to 'Yes' discomfort on the binary scale was taken as BCD (borderline between comfort and discomfort) value for that participant. If the borderline value was lower than or equal to the middle level intensity (scene 10\%), the participant was categorized as less sensitive to glare and if it was higher than mid-intensity than the participant was categorized as more sensitive to glare.

The MPOD distribution for OD (right eye) and OS (left eye) is reported as density plots. For those participants where the pigment density was different in OD and OS, we took the minimum of the two values for the analysis. We applied Wilcoxon rank-sum test (Wilcoxon, 1945) for comparing two independent non-parametric samples to determine if there is a significant difference at $\alpha=0.05$ in the MPOD levels between the less and more sensitive groups.

\section{Results}

The analysis outcomes for the 53 participants showing consistency in their ratings are presented in the following subsections.

\subsection{Subjective responses to glare}

Subjective responses to glare, as reported by the participants, were analyzed for each of the experimental conditions listed in Table 1. The resulting distribution of glare votes for each glare level is shown in Figure 4 for the binary glare scale and in Figure 5 for the 6-pt glare scale.

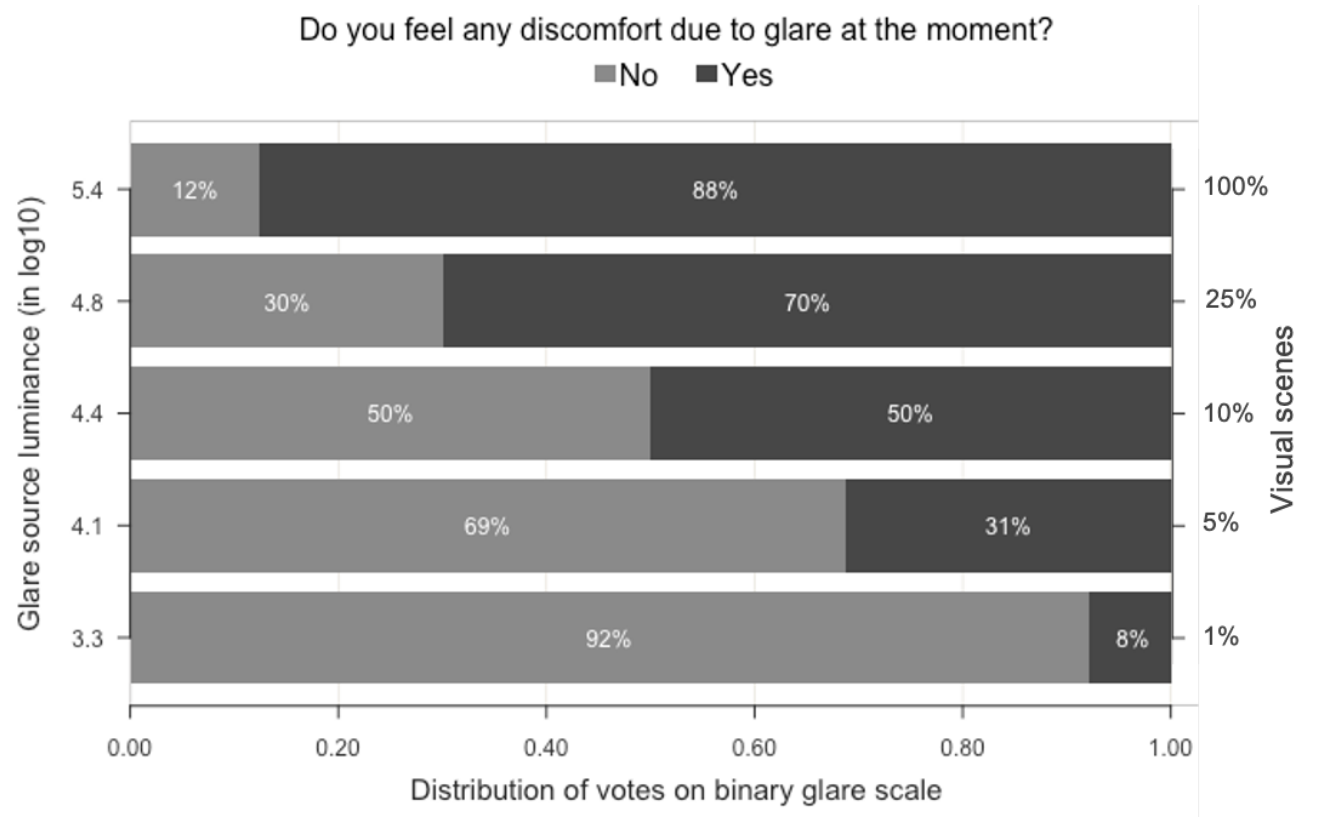

Figure 4 Distribution of participant's response to glare levels on binary glare scale

In general, the subjective glare responses follow the glare source luminance. Increase in luminance increases the discomfort glare perceived by the participants, thereby validating the experimental protocol followed. Similar trends can be observed in the responses on both scales in Figure 4 and Figure 5. However, the threshold between discomfort and comfort tends to get lower with the 6-pt scale compared to the binary scale: even if participants experienced a slight discomfort, they would tend to report no discomfort on the binary scale. This can be due to the finer classification of the scale and the semantic differences in the scale labels. Of all the 
participants who voted "Slight discomfort" on the 6-pt glare scale, 53\% had voted "Yes" on the binary scale. Since binary scale provides a clearer semantic distinction between comfort and discomfort, we decided to derive the glare thresholds for each participant based on the glare voting done on the binary glare scale. The Spearman's rank correlation coefficient between the glare source luminance and the subjective responses reported on 6-pt scale was 0.71 , which is indicative of a strong effect size following Cohen's standard (Cohen, 1988).

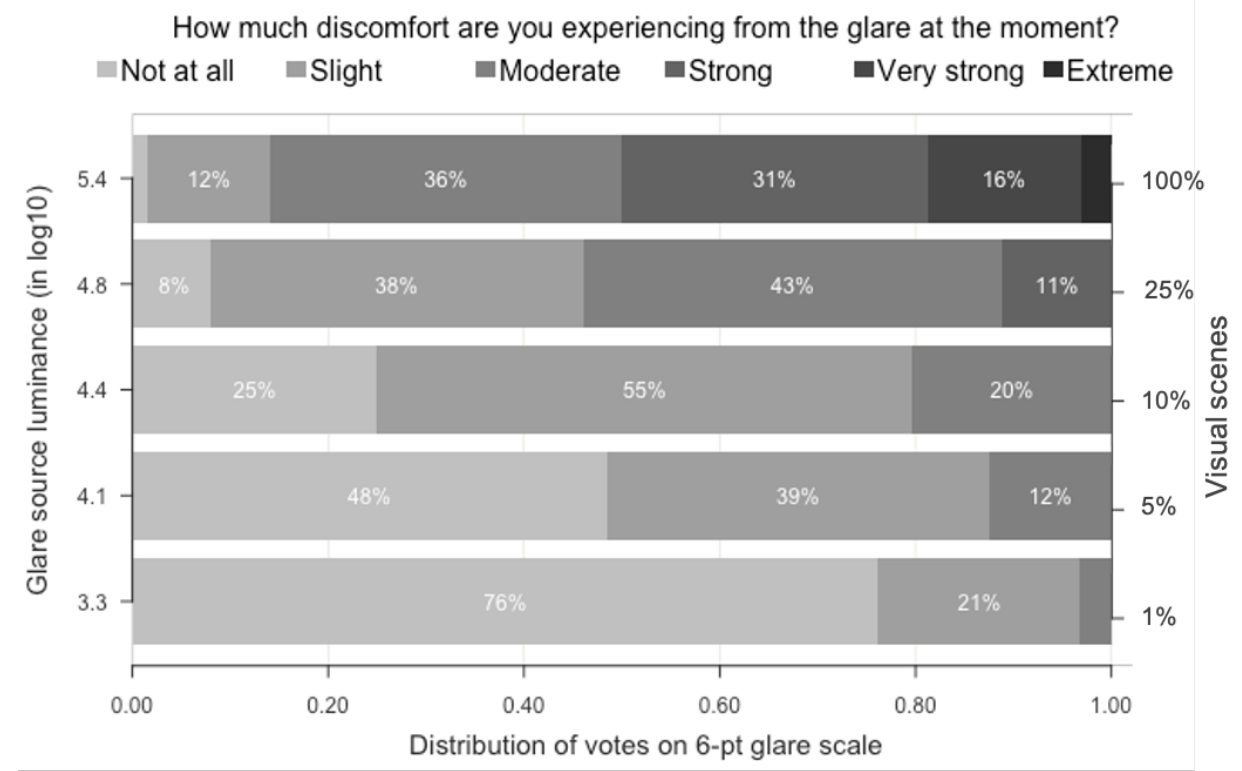

Figure 5 Distribution of participant's response to glare levels on 6-pt scale glare scale

\subsection{Glare thresholds}

Based on the participants' responses to glare on the binary scale, we derived glare thresholds for each participant, i.e., their borderline values between comfort and discomfort. As shown in Figure 6, the resulting discomfort glare thresholds are well distributed across the range of light intensity shown to the participants, which indicates quite some inter-individual variability in comfort perception. The glare thresholds obtained from the sensitivity test were in agreement with those obtained from the post-trial session (Pearson's correlation coefficient $\rho=0.61$ ). Based on the glare level categorization rules described in section 2.3 , we found that $47 \%$ of the participants to be less sensitive and $53 \%$ more sensitive to glare in our sample.

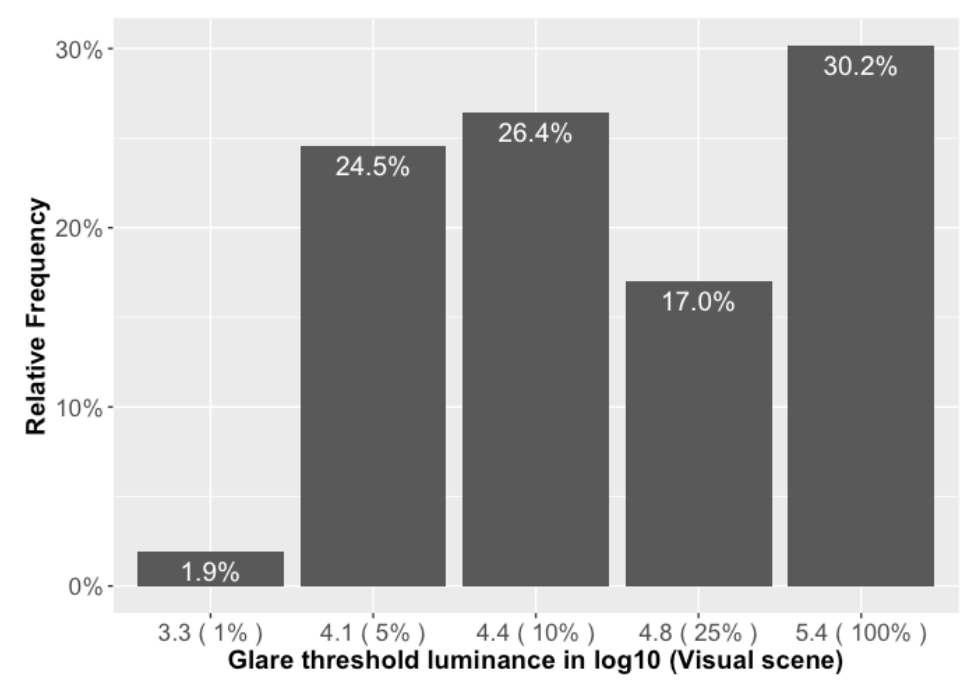

Figure 6 Distribution of participant's glare threshold luminance values 


\subsection{MPOD and glare sensitivity}

The MPOD levels among the sample were found to range from 0.14 to 0.86 , with mean MPOD measurements for OD and OS of 0.49 and 0.50 . The correlation coefficient of mean MPOD measurements of OD and OS was 0.87 (Pearson correlation coefficient, $P<0.0001$ ), indicating excellent inter-eye predictability of the measurement device. Figure 7 presents the distribution of the MPOD for the left and the right eye which follows a normal distribution (Shapiro-Wilk test, $P=0.32$ and 0.06 ). We selected the minimum of OS and OD as an indicator of MPOD for a participant for the analysis purpose.

We then compared the mean differences in MP levels between the two groups of the participants categorized as less and more sensitive to glare by applying a Wilcoxon rank-sum test on the two groups (Figure 8 ). We found statistically significant differences in MPOD between the two groups at $\alpha=0.05$ (Wilcoxon test $p=0.028$ with small effect size, $\rho=0.30$ ), which shows that further investigation on MP would seem promising to provide new insights on the factors influencing glare perception. Figure 8 shows the boxplots of the two groups together with the mean values of MPOD: MP levels were actually found to be slightly higher in the more sensitive group compared to less sensitive groups, which contradicted our expectation and warrants further investigation.
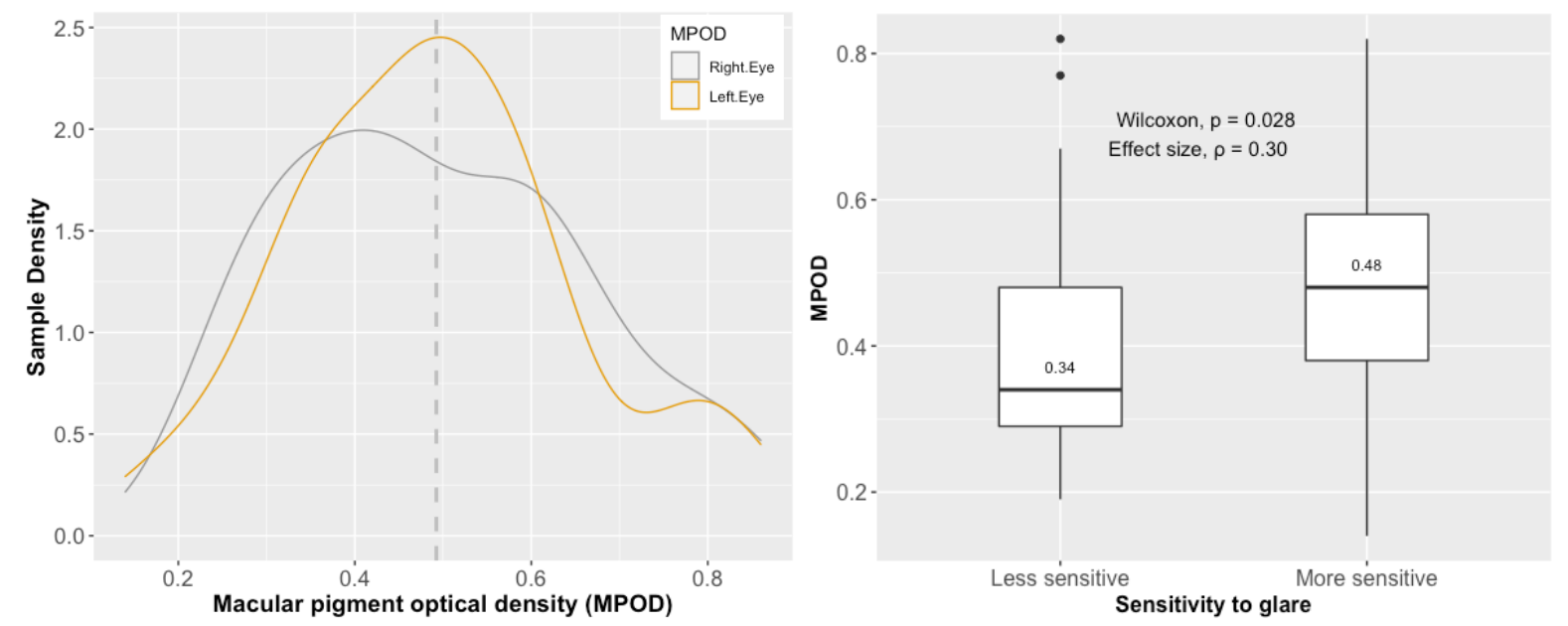

Figure 7 (left) Distribution macular pigment optical density in the sample population

Figure 8 (right) Comparison of MPOD values in the two groups less sensitive and more sensitive to glare

\section{Conclusion and discussion}

This study contributes to the present knowledge on discomfort glare sensitivity and its relationship with macular pigment. In addition, this study provides a methodological approach to quantify individual glare sensitivity thresholds. The method was found to be consistent in evaluating within-subject responses.

In this study, we evaluated a person's sensitivity to glare and compared it with their measured MPOD levels. MPOD levels were found to be statistically different between the two groups of participants (less vs. more sensitive to glare) but with a small effect size. We found slightly lower MPOD in the group having higher glare thresholds: this is surprising since it contradicts the previous studies. One explanation could be that most of the previous studies were conducted under disability glare levels and the glare source was visible within foveal range. However, in this study we have discomfort glare scenarios with glare source visible outside the fovea. Since macula covers about $6.7^{\circ}$ in the horizontal and $3.0^{\circ}$ in the vertical directions of the central retina, its influence is likely to decline towards the perifoveal light sources that occurs in our experimental scenarios.

The limitations of the study also have an impact on the accuracy and the generalizability of the results. In our experiment, the exposure to glare conditions was very short-term and subjective 
assessment of glare can vary with longer exposure time. Previous studies give us indications about the variation in average macular pigment density as per the ethnicities of the sample, for example, Davey and authors (Davey et al., 2020) found higher pigment density in south Asian Indians and Hispanics compared to Caucasians and African Americans. However, in our study, we didn't control for ethnicity of the participants and due to small sample size, we assumed the same averages across the recruited participants of different ethnicities. Additional data is needed to validate the finding and more in-depth analyses are required to confirm the results obtained in this preliminary analysis.

In the next steps of the study, in collaboration with Hospital Ophthalmic Jules-Gonin, we will be employing physical method of Fundus autofluorescence imaging to examine the macula. We will also measure the photostress recovery time in our sample, which is closely linked to macular pigment and glare sensitivity. We will extend the protocol to daylit scenarios and increase the exposure time to the experimental conditions to better replicate the typical office conditions.

\section{Acknowledgement}

This study was conducted within the framework of a broader collaboration with the Hospital Ophthalmic Jules-Gonin and the authors would like to thank Prof. Aki Kawasaki and Prof. Chiara Eandi for their insights on these initial results. The work is funded by Swiss National Foundation project (SNF) grant for the project "Visual comfort without borders: interactions on discomfort glare" number 200020_182151.

\section{References}

Bach, M., 1996. The Freiburg Visual Acuity test--automatic measurement of visual acuity. Optom Vis Sci 73, 49-53. https://doi.org/10.1097/00006324-199601000-00008

Bernstein, P.S., Delori, F.C., Richer, S., van Kuijk, F.J.M., Wenzel, A.J., 2010. The value of measurement of macular carotenoid pigment optical densities and distributions in agerelated macular degeneration and other retinal disorders. Vision Research 50, 716-728. https://doi.org/10.1016/j.visres.2009.10.014

Carlucci, S., Causone, F., De Rosa, F., Pagliano, L., 2015. A review of indices for assessing visual comfort with a view to their use in optimization processes to support building integrated design. Renewable and Sustainable Energy Reviews 47, 1016-1033. https://doi.org/10.1016/j.rser.2015.03.062

Cohen, J., 1988. Statistical Power Analysis for the Behavioral Sciences. Routledge, New York. https://doi.org/10.4324/9780203771587

Davey, P.G., Lievens, C., Amonoo-Monney, S., 2020. Differences in macular pigment optical density across four ethnicities: a comparative study. Ophthalmol Eye Dis 12, 251584142092416. https://doi.org/10.1177/2515841420924167

Delori, F.C., Goger, D.G., Hammond, B.R., Snodderly, D.M., Burns, S.A., 2001. Macular pigment density measured by autofluorescence spectrometry: comparison with reflectometry and heterochromatic flicker photometry. J Opt Soc Am A Opt Image Sci Vis 18, 1212-1230. https://doi.org/10.1364/josaa.18.001212

Eble-Hankins, M.L., Waters, C.E., 2005. VCP and UGR Glare Evaluation Systems: A Look Back and a Way Forward. LEUKOS 1, 7-38. https://doi.org/10.1582/leukos.2004.01.02.001

Fotios, S., 2009. Research Methods to Avoid Bias in Categorical Ratings of Brightness. Leukos 5, 167-181. https://doi.org/10.1582/leukos.2008.05.03.002

Lien, E.L., Hammond, B.R., 2011. Nutritional influences on visual development and function. Progress in Retinal and Eye Research 30, 188-203. https://doi.org/10.1016/j.preteyeres.2011.01.001

Loughman, J., Akkali, M.C., Beatty, S., Scanlon, G., Davison, P.A., O'Dwyer, V., Cantwell, T., Major, P., Stack, J., Nolan, J.M., 2010. The relationship between macular pigment and visual performance. Vision Research 50, 1249-1256. https://doi.org/10.1016/j.visres.2010.04.009

Stringham, J.M., Bovier, E.R., Wong, J.C., Hammond, Jr, B.R., 2010. The Influence of Dietary Lutein and Zeaxanthin on Visual Performance. Journal of Food Science 75, R24-R29. https://doi.org/10.1111/j.1750-3841.2009.01447.x

Stringham, J.M., Fuld, K., Wenzel, A.J., 2004. Spatial Properties of Photophobia. Invest. Ophthalmol. Vis. Sci. 45, 3838. https://doi.org/10.1167/iovs.04-0038 
Stringham, J.M., Garcia, P.V., Smith, P.A., McLin, L.N., Foutch, B.K., 2011. Macular Pigment and Visual Performance in Glare: Benefits for Photostress Recovery, Disability Glare, and Visual Discomfort. Invest. Ophthalmol. Vis. Sci. 52, 7406. https://doi.org/10.1167/iovs.10-6699

van der Veen, R.L.P., Berendschot, T.T.J.M., Hendrikse, F., Carden, D., Makridaki, M., Murray, I.J., 2009. A new desktop instrument for measuring macular pigment optical density based on a novel technique for setting flicker thresholds. Ophthalmic and Physiological Optics 29, 127-137. https://doi.org/10.1111/j.1475-1313.2008.00618.x

Wenzel, A.J., Fuld, K., Stringham, J.M., Curran-Celentano, J., 2006. Macular pigment optical density and photophobia light threshold. Vision Research 46, 4615-4622. https://doi.org/10.1016/j.visres.2006.09.019

Wilcoxon, F., 1945. Individual Comparisons by Ranking Methods. Biometrics Bulletin 1, 80-83. https://doi.org/10.2307/3001968 